\title{
A Study on Information Technology Freelancer Matching with Exploiting Blockchain in Gig Economy
}

\author{
${ }^{1} \mathrm{JINHO}$ LIM, ${ }^{2} \mathrm{KW}$ ANSIK NA, ${ }^{3}$ SEUNGCHEON KIM \\ ${ }^{1}$ Doctoral Student, Dept. of Smart Convergence Consulting, Hansung University, \\ Seoul, KOREA
}
${ }^{2}$ Corresponding Author Professor, Dept. of Management Information System, Seowon University, KOREA
${ }^{3}$ Corresponding Author Professor, Dept. Of IT Convergence Eng.,Hansung University, Seoul, KOREA

\begin{abstract}
In this paper, we propose a freelancer matching of a recommended recruitment system in a situation in which the freelance type employment market defined by peer-to-peer transactions, mutual evaluation of freelancers and clients, time flexibility of service providers, and the use of service providers' tools and assets are expanding. In order to increase the reliability and accuracy of recommendation through reputation, we propose a reputation ranking technique for reputation system, which is a kind of personalized recommendation system, based on the blockchain technology. We propose a reputation system model suitable for recruitment matching service. We have aims to study the method of implicitly extracting user reputation information based on two factors suitable for word of mouth among information source reliability factors. In other words, this paper defines a method for automatically extracting two reliability factors of freelancers from past reputation information, and proposes a method for effectively predicting freelancer applicant' reputation information using only the information of high-reliability evaluators.
\end{abstract}

Keywords: IT freelancer, Job Matching, Career Management, blockchain, Career Verification

Received: August 8, 2020. Revised: January 20, 2021. Accepted: February 5, 2021. Published: February 12, 2021.

\section{Introduction}

The Gig Economy, which is defined as $\mathrm{P} 2 \mathrm{P}$ (Peer to Peer) transactions using Information Technology devices, mutual evaluation between freelancer job seekers and recruiters. In addition, according to Staffing Industry Analyst' report dated on January 2014 "Online Staffing" Platform Business - Industry Segment Forecast Through 2020", the global online job market is expected to grow from $\$ 16$ billion in 2014 to $\$ 46$ billion in 2020 to $\$ 5$ billion per year. While the growth of gig economy and online matching platform has expected the opportunities for matching to Information Technology freelancers, there are still problem to address such as followings.

Firstly, centralization of information ownership means Information Technology freelancers have no information of their own information nor are they compensated for information submitted to clients.
Secondly, required verification means there always remains the potential risk that anyone easily tamper with his/her skill or career, which causes unfair competition for limited matching opportunities suggested by interested clients.

Thirdly, imposition of brokerage fees means if you are going through a recruiting platform or headhunter to obtain information about clients and projects, you will incur an intermediary fee, which will reduce your income.

Fourthly, contract and project quality means even if the parties have agreed on the scope and duration of the project as well as the payment period, Information Technology freelancers may face a problem of delay in payment and clients be concerned about project quality [1]. 


\section{Related Work}

\subsection{Online Reputation System and Collaborative Filtering technique}

An online reputation system is a software system that collects feedback information of online transactions to calculate the trader's reputation to support future job matching decisions for online job seekers. Analyzing existing online reputation system research, reputation system can be classified according to how to collect reputation information, reputation scoring and ranking method, and feedback information management method. However, in this study, the concept and classification method of reputation system will be largely defined in terms of technical aspects based on "user reputation information collection method" and "item reputation information collection method" [2][3]. Collaborative filtering is a way of recommending items that users with similar tastes prefer. This presupposes that users who gave similar ratings to the same factors would give similar ratings to new factors.

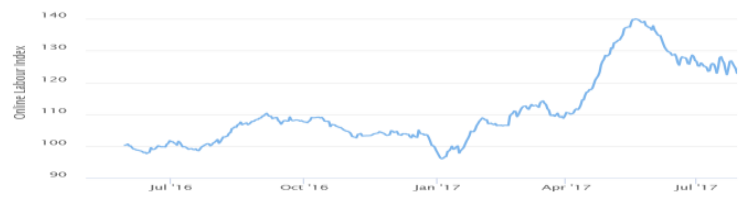

Table 1. Online Labor Index Trend (2017)

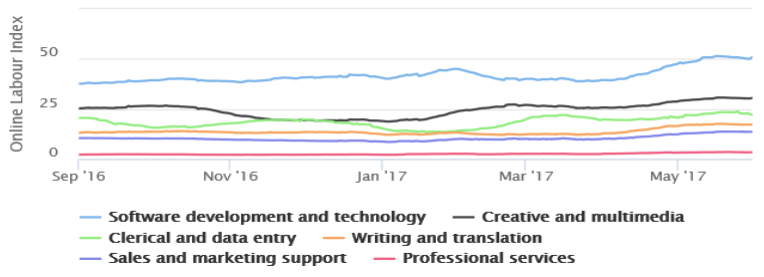

Table 2. Online Labor Index and Occupation(2017)

Table 1 and Table 2 shows IT freelancer will be increased as the meaning of job, according to the Online Labor Index developed by the University of Oxford in the UK, the online labor index grew to $26 \%$ in 2017 (as of July) compared to 2016, and the Information Technology freelancer activity in the SW / IT development sector was 30\% high. UpWork, the world's largest Information Technology freelancer recruitment platform, analyzed $20 \%$ of the fastest growing technologies are related to digital technology. In the Q1, 2018 Skills Index report, the top 20 of the hottest skills in the U.S. freelance job market show numerous Information Technology technologies including Blockchain, Google Cloud Platform, Google App Engine API, GitLab, Go development, Apple UIKit, Apple Xcode, and Node.js. According to the report, more than $59 \%$ of HR managers used Information
Technology freelancers in 2017 and $57 \%$ of respondents said they will hire more Information Technology freelancers over the next 10 years as above mentioned Table 1 and Table 2 as proved the trend.[4]

\subsection{Collaborative Filtering and Information Source Reliability}

The traditional collaborative filtering technique is to automate the Word of Mouth (WoM) between the nominee (client) and the recommender. In this case, the recommender plays the role of an information source for providing information, and accordingly, the recommendation quality of the collaborative filtering technique predicts how credible the information source is for the recommender. It can be formed as a predictor group '[10]. In traditional collaborative filtering, to provide job seekers with 'personalized' recommendations, they form a referral group called 'Neighbors' based on the similarity between the target job seekers and past matching patterns, and present their opinions to the target job seekers. This technique is called similarity-based collaborative filtering. However, this similarity-based collaborative filtering has traditionally been difficult to find 'neighbors' with target job seekers, such as data sparsity problems, cold start problems, etc. In addition to the problems that are pointed out, there is a predictor reliability problem. In this paper, we look at collaborative filtering in terms of information source reliability and extract a group of evaluators based on two reliability criteria with exploiting blockchain technology.[3]

\subsection{Cryptocurrencies in blockchain technology}

As well-known, blockchain is a decentralized ledger, concurrent transaction and data management solution, especially, cryptocurrencies is the widely already adoption of transactions tools and cross-border payments in finance sector. Since, blockchain technology is getting embedded in the e-commerce services, the cryptocurrencies are gaining huge prevalence.

\section{Methodology}

The Information Technology freelancer job matching plate system model proposed in this paper targets the P2P(Peer to Peer) model which is an interactive plate decision structure. Based on the source credibility theory, two reliability criteria will be selected separately. [Fig1] shows reputation flow between Information Technology freelancers and client as reputation index with categorizing quantitative factors and qualitative factors. We will call the online matching 
platform as Career View(CV as abbreviation) is a blockchain-based Information Technology freelancer matching platform that creates a proven competencybased transparent and secure Information Technology freelancer matching ecosystem through decentralized verification protocols.[4]

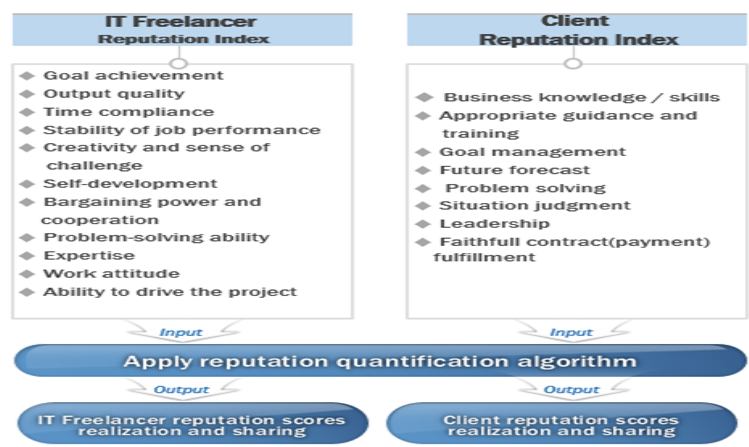

Figure 1. Reputation Quantifying

Fig1 shows Career View, Information Technology freelancer matching system, consists of several core components Information Technology freelancer competency verification and matching, Information Technology freelancer and client safe transaction, reputation management system. To achieve these features, the matching system should be distributed, decision process be transparent and there should be a clear reward system for platform scalability and user motivation.

\subsection{Decentralized Verification Protocol}

Information Technology freelancers have the right to access to profile management, reputation and recommendation management via a CV(Career View) vault. Registered Information Technology freelancers manage their own abilities and skills and find opportunities to participate in new projects easily. In addition, they can share relevant information with another Information Technology freelancer who is regarded as suitable for the project. In CV(Career View) vault, Information Technology Freelancers can keep the relevant certificate and a variety of materials to demonstrate their ability and skills. CV(Career View) vault runs on P2P-based, decentralized, distributed consensus and secure data storage systems. Ethereumbased blockchains are only used to hold the address of the data.

Information Technology freelancers are the only ones who own their professional reputation. CV(Career View) vault complies with GDPR (General Data Protection Regulations) and can modify, delete, change and update related data. Decentralized verification protocol means
Career View builds a recruitment service ecosystem based on trust between Information Technology freelancers and client by verifying the education, career and skill of Information Technology freelancers through a decentralized protocol and quantifying reputation scores. Information Technology freelancers will be a self-information entity to manage and archive the information verified by Career View protocol. Managing reputation scores enable both Information Technology freelancers and clients to better participate in projects and produce results.

Career View builds a recruitment service ecosystem based on trust between Information Technology freelancers and clients by verifying the education, career and skill of Information Technology freelancers through a decentralized protocol and quantifying reputation scores. Information Technology freelancers will be a self-information entity to manage and archive the information verified by Career View protocol. Managing reputation scores enable both Information Technology freelancers and clients to be better participants in projects and produce results. [Fig2] shows Information Technology Freelancer Job matching structure as above mentioned logical framework.[5][6]

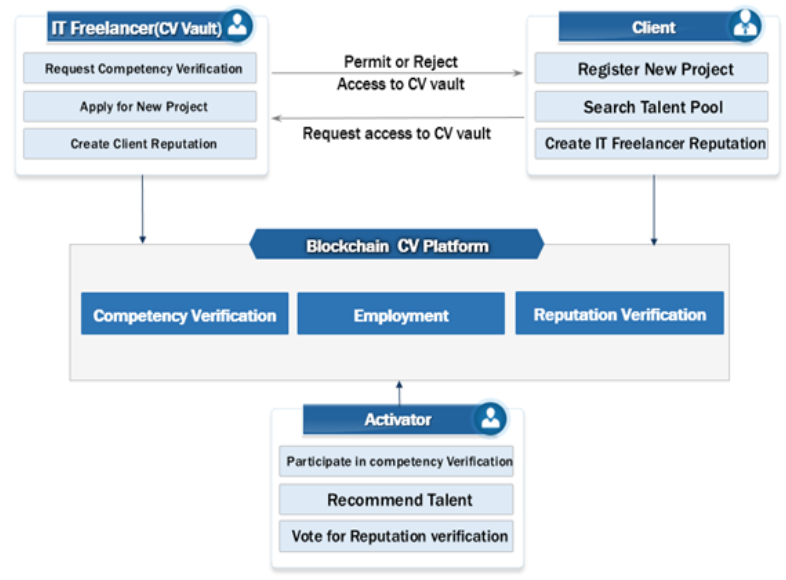

Figure 2. Freelancer Job Matching structure.

\subsection{Smart Contract Based on Matching Contracts and Rewards}

When the matching contract between Information Technology freelancer and client is made, the matching contract details are stored in the block and kept unchanged. Once the project is completed and an agreement has been reached on the reputation of Information Technology freelancer and project quality, compensation will be done immediately by the smart contract. At this time, all activators involved in the reputation and consensus will be rewarded. If a dispute 
arises between the parties, it will be passed to the voting process and compensation will be delayed until the dispute is settled as showing Fig 3.

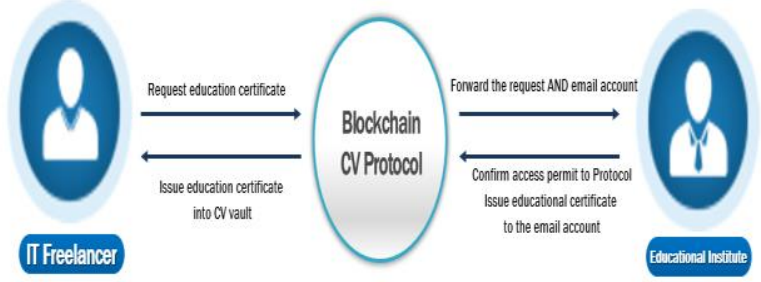

Figure3. Career Verification Process

Fig 3 and Fig 4 shows reputation score in Career View play a key role in the decision-making process. The reputation score of the core players is obtained from mutual evaluation between the Information Technology freelancer and client after completion of the project, skill verification of the Information Technology freelancer, and participation in voting to solve dispute. It is an essential component to create a reliable and secure Career View service ecosystem. As a utility token, View token is a means of compensating for activities in the Career View service, such as CV(Career View) vault management, information access, reputation creation, and voting. View token should be saved as deposit when participating in each activity. When it is judged to be a good reputation activity, the reputation score will go up, otherwise the token will not be recovered and the reputation score will go down. An objective, fairly earned reputation score increases the opportunity for Information Technology freelancers to participate in more projects and the opportunity for clients to hire the right talent with the best profile matching their project. Career View will bring fundamental changes to matching methodology providing competency-based services and smart contract-based hiring practices and will secure clear compensation for parties' efforts with reward ecosystem of View Toke as an utility token. [4][5].

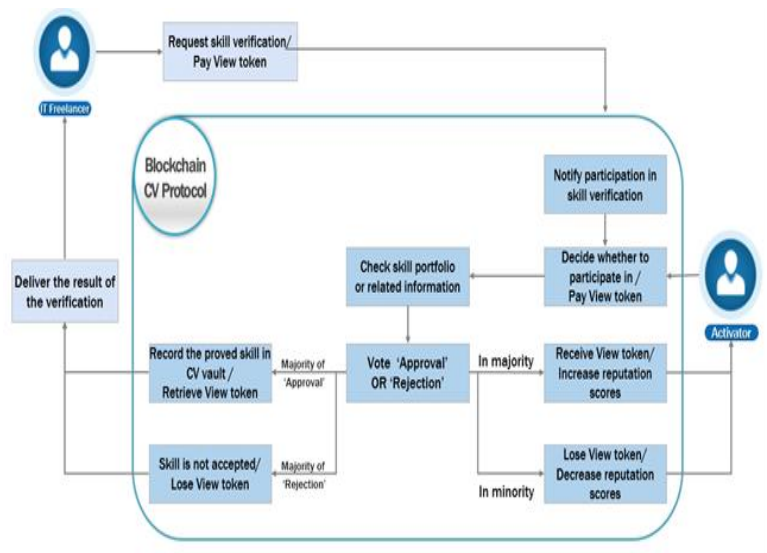

Figure 4. Skill Verification Process

\subsection{Reputation Score Quantifying, Realization and Sharing}

Reputation is quantified on a basis of the activities of all core users, including Information Technology freelancer resume writing, Information Technology freelancer competency verification, project support, matching, Information Technology freelancer - client mutual evaluation after project completion and Information Technology freelancer recommendation. If the score is entered according to each reputation index, reputation quantifying is conducted by using normalization of preference by type, reputation, relevance scale and text mining with $\mathrm{R}$ (statistical package) according to service reputation quantification algorithm. Machine learning can be applied to overcome the limitations of algorithms that are interpreted literally and dedicated to a specific purpose. Machine learning can indirectly learn discrimination, negative evaluation, and implicit bias through data. In addition, it can overcome the limitation of a centralized reputation system (a system that has a separate server to store and manage user's reputation and transaction records. As the server makes the reputation information available by obtaining and disclosing it, the participants can check the reputation of each other through the server when determining whether to perform the transaction of the specific user) and use the distributed reputation system with P2P network environment such as Napster Reputation system (There is no central server for reputation management. Users' transaction records and reputation values are recorded in a distributed manner. Individual users participating in the service use DHT (Distributed Hash Table) to store other users' reputation information and provide it when the user requests it. In this way, the reputation information requestor and providers do not know each other) [4][5]. Following Fig 4 shows comparison 
between centralized reputation system and decentralized reputation system.

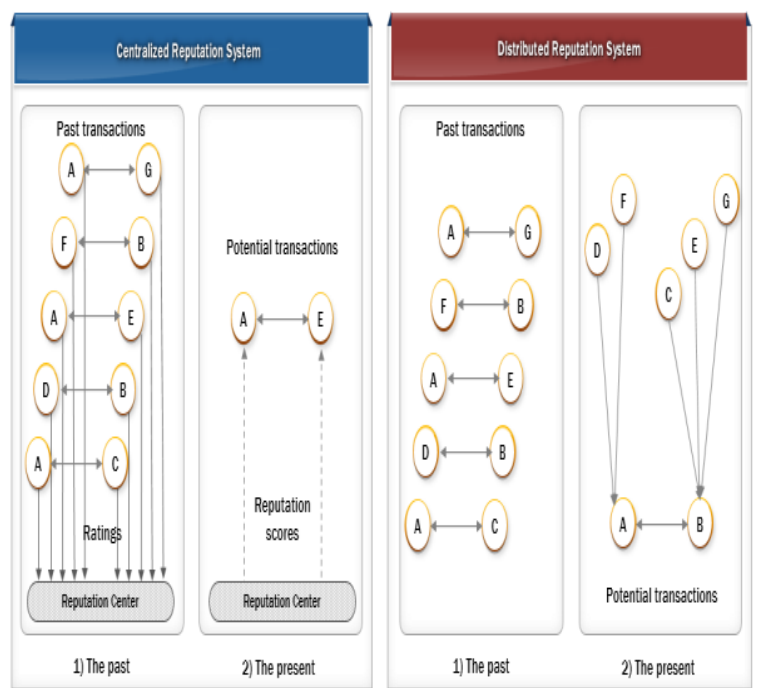

Figure 5. Centralized reputation system and distributed reputation system

Smart contract-based matching procedures for Information Technology freelancers are conducted through project registration, researching the talent pool, matching the best profiles, Information Technology freelancer recommendation, both Information Technology freelancer and client evaluation after project completion. Fig 5 shows the matching procedure as logical framework.[6][7].

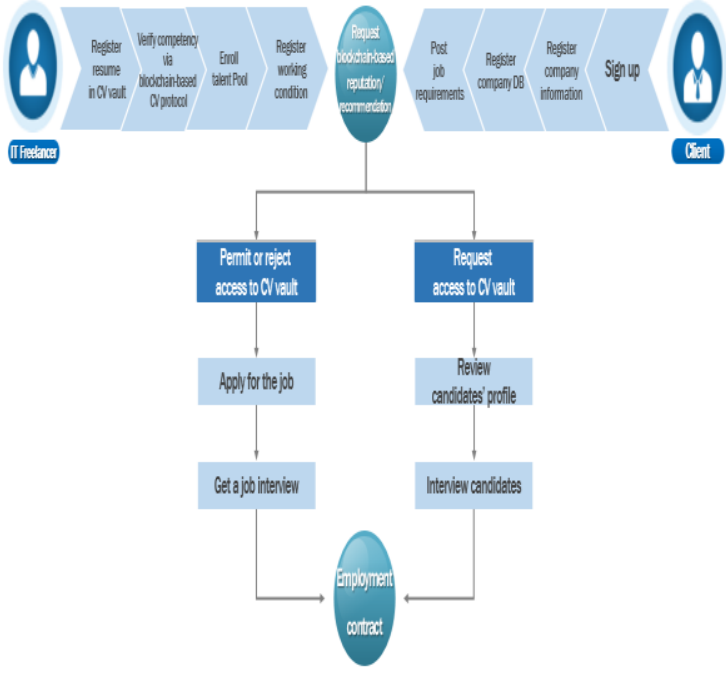

Figure 6. Project registration and Information

Technology freelancer matching procedure After the project is completed, Information Technology freelancer and client are mutually assessed to improve service reliability. Client will evaluate the competency and skills of Information Technology freelancers based on the tasks performed by Information Technology freelancers. Information Technology freelancer conducts an evaluation of clients who hired them for the project execution. The artificial intelligence algorithm applied to the Career View platform provides projectrelated evaluation factors. Information Technology Freelancer Ranking based on Professional level. Information Technology freelancer ranking is determined by the competency score obtained through the verification process and the reputation score obtained from the project activity as following Fig 7.

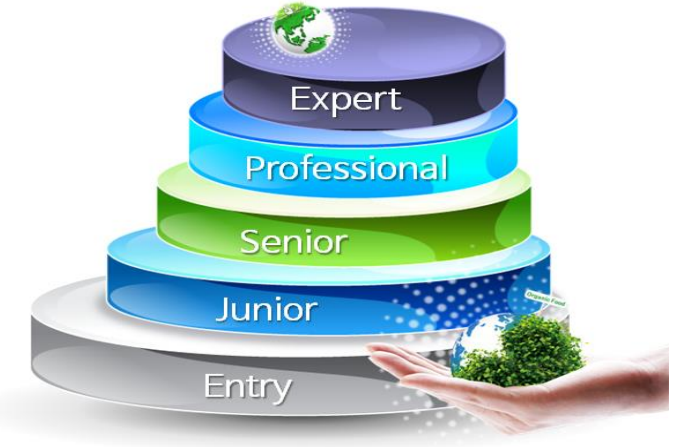

Figure 7. Information Technology Freelancer Ranking based on Professional level

As above Fig 7 mentioned Information Technology freelancer ranking level to clarify criteria, we assume Ranking of Entry means Information Technology career of 1-3 years or less, ability to understand fundamental and overall procedures with basic skill. Ranking of Junior means Information Technology career for $4 \sim 7$ years or less, ability to take leadership in challenging areas or specific task with practical skills. Ranking of Senior means Information Technology career for 8 to 10 years or less, ability to make comprehensive decisions in a certain area with a wide range of expertise and practical skills. Ranking of Senior means Information Technology career for 8 to 10 years or less, ability to make comprehensive decisions in a certain area with a wide range of expertise and practical skills. Ranking of professional means Information Technology career for 11 to 15 years, ability to make important decisions and guide projects in complex and challenging areas with a wide range of expertise and skill. Ranking of Expert means Information Technology career for over 15 years, ability to propose a solution that has significant effect on project completion in complex and challenging areas with a vast expertise and skills

Also, we assume client ranking as project size as following Fig 8 to shows client ranking based on project size. From this kind of category, Clients are ranked according to the size of the project to be conducted. 


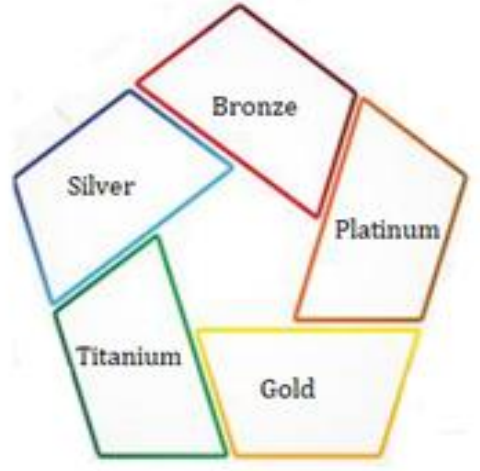

Figure 8. Client ranking based on project size

We put the assumption criteria as Ranking of Bronze means Less than $\$ 9$ thousand to $\$ 27$ thousand of project budget, less than 1 month of working period, less than 3 people of team member. Ranking of Silver means Less than $\$ 27$ thousand to $\$ 45$ thousand of project budget, less than 1 month to 3 months of working period, 3 and 4 people of team member. Ranking of Titanium means Less than $\$ 45$ thousand to $\$ 90$ thousand of project budget, less than 3 to 6 months of working period, 5 and 7 people of team member. Ranking of Gold means less than $\$ 90$ thousand to $\$ 270$ thousand of project budget, less than 6 months to 1 year of working period, 7 and 9 people of team member. Ranking of Platinum means More than $\$ 270$ thousand of project budget, more than 1 years of working period, more than 10 people of team member [8][9].

\section{Conclusion}

In this paper, We propose, Career View is an advanced Career PASS utilizing blockchain technology. Career PASS is developed to serve to college student the integrated qualification management and job recommendation solutions. Career PASS has been established in more than 30 major universities in Korea and helped college students begin their successful career path by optimizing the integration of personalized personal history management and recommendation algorithms. Beyond countries and regions does Career View based on blockchain seek to create an ecosystem specializing in the Information Technology freelancer job market where Information Technology freelancer matching, reputation, talent search, client evaluation, community and business relations management services are provided. In this study, we propose a ranking method for reputation system, which is a kind of personalized recommendation system based on blockchain technology, and present a Information Technology freelance job matching system. In the future research, there is a need for additional verification so that the experiment and performance verification of the plate system model design proposed in this study can be performed [10]. For the reason, this study will contribute to provide opportunities to enhance Information Technology freelancer information and ownership reward for use of their information by solving those existing problems with the blockchain technology which is attracting attention as an innovative technology that will change the freelancer matching system. Especially, when we use information on the Internet, we face the challenge of judging and choosing the right ones from a lot of information. This is linked to the problem of 'what information is correct?', and the solution to this problem is the 'reputation system' . Since online feedback refers to the evaluation of other participants about the items which they wish to provide, the reputation system may be classified as a kind of recommender system

\section{References}

[1] Arunima Ghosh, Shashank Gupta, Amit Dua, Neeraj Kumar, "Security of Cryptocurrencies in blockchain technology: State-of-art, challenges and future prospects, Journal of Network and Computer Application, Volume 163, 2020

[2] Thomas Conlon, Shaen Corbet, Richard J. McGee, "Are cryptocurrencies a safe haven for equity markets? An international perspective from the COVID-19 pandemic, Research in international Business and Finance, Volume 54, 2020

[3] Lanouar Charfedding, Noureddine Benlagha, Youcef Maouchi, "Investigating the dynamic relationship between cryptocurrencies and conventional assets: Implications for financial investors", Economic Modelling, Volume 85, 2020

[4] Dehua Shen, Andrew Urquhart, Pengfei Wang, "A three-factor pricing model for cyrptocurrencies", Finance Research Letters, Volume 34, 2020

[5] Alexander and Dakos, "A Critical Investigation of Cryptocurrency Data and Analysis", Quant. Finance, 20(2) (2020), pp.173-188.

[6] Conlon and McGee, "Safe Haven or Risky Hazard? Bitcoin during the Covid-19 Bear Market", 2020

[7] Mensi et al, "Doest Bitcoin co-move and share risk with Sukuk and world and regional Islamic stock markets? Evidence using a time-frequency 
approach”, Res. Int. Bus. Finance, 2020

[8] Wang, Shen, Zhang "How does economic policy uncertainty affect the Bitcoin market?”, 2020

[9] Giancarlo Giudici, Alistair Milne \& Dmitri Vinogradov, "Cryptocurrencies: market analysis and perspective", Journal of Industrial and Business Economics, 2019

[10] Jinho Lim, Kwansik Na, Yenyoo You, "The Architecture Design of Students' Career Information Management System Using Blockchin Technolgoy, IJITEE,ISSN:2278-3075, 2019
Creative Commons Attribution License 4.0 (Attribution 4.0 International, CC BY 4.0)

This article is published under the terms of the Creative Commons Attribution License 4.0

https://creativecommons.org/licenses/by/4.0/deed.en_US 